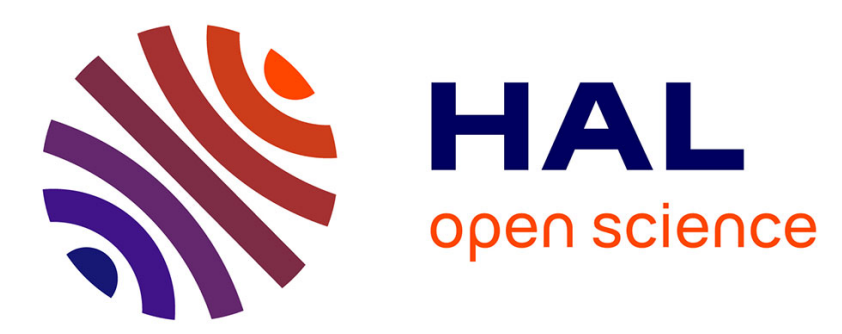

\title{
In search of the holy grail: Looking for the weakest failure detector for wait-free set agreement
}

\author{
Michel Raynal, Corentin Travers
}

\section{To cite this version:}

Michel Raynal, Corentin Travers. In search of the holy grail: Looking for the weakest failure detector for wait-free set agreement. [Research Report] PI 1811, 2006, pp.14. inria-00089977

\section{HAL Id: inria-00089977 \\ https://hal.inria.fr/inria-00089977}

Submitted on 25 Aug 2006

HAL is a multi-disciplinary open access archive for the deposit and dissemination of scientific research documents, whether they are published or not. The documents may come from teaching and research institutions in France or abroad, or from public or private research centers.
L'archive ouverte pluridisciplinaire HAL, est destinée au dépôt et à la diffusion de documents scientifiques de niveau recherche, publiés ou non, émanant des établissements d'enseignement et de recherche français ou étrangers, des laboratoires publics ou privés. 


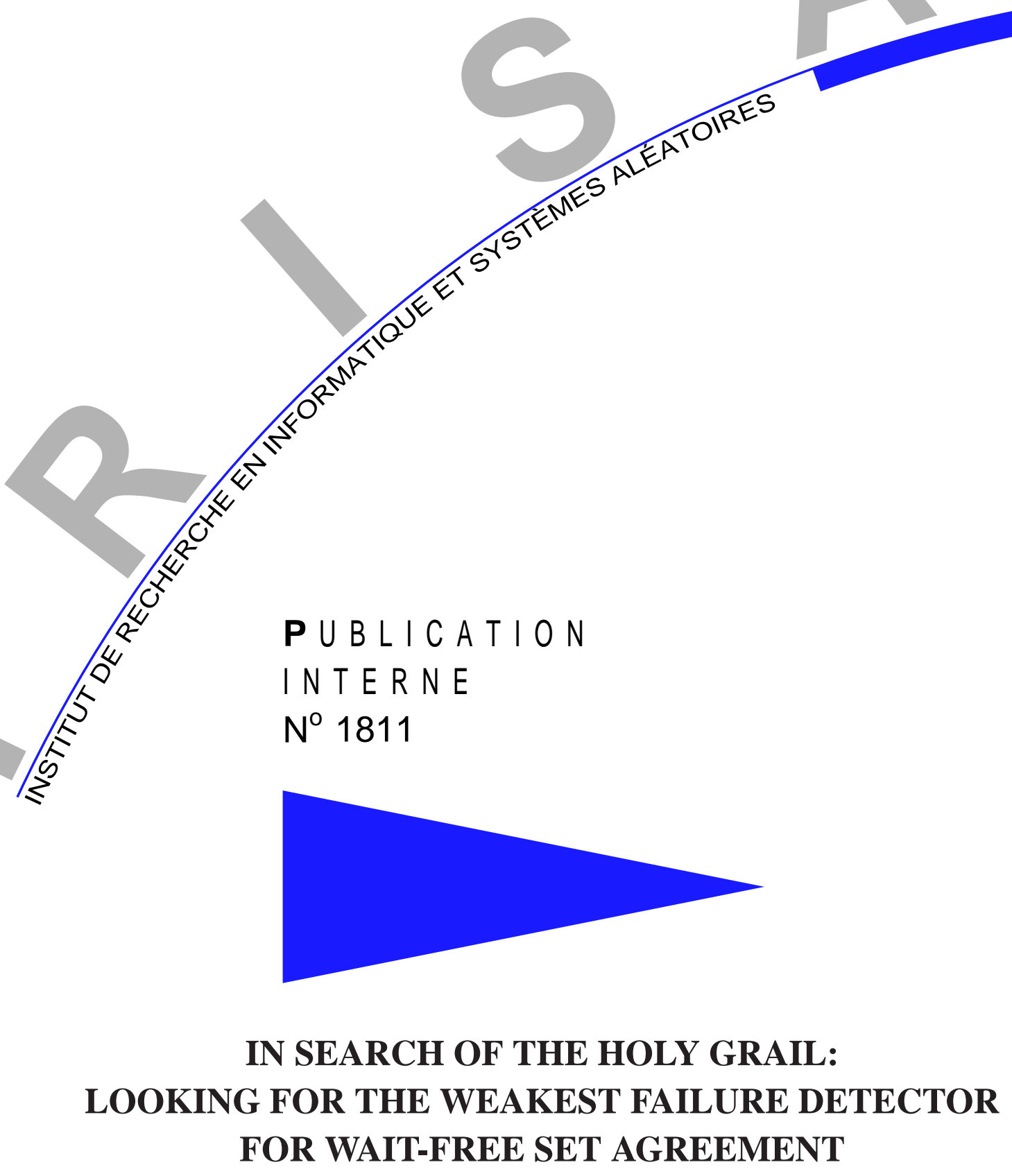

MICHEL RAYNAL CORENTIN TRAVERS 



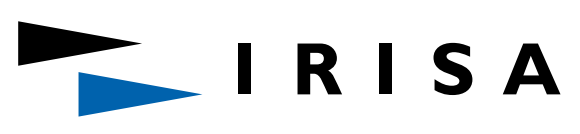

\title{
In search of the holy grail: Looking for the weakest failure detector for wait-free set agreement
}

\author{
Michel Raynal ${ }^{*} \quad$ Corentin Travers ${ }^{* *}$ \\ Systèmes communicants \\ Publication interne $\mathrm{n}^{\circ} 1811$ - Juillet $2006-14$ pages
}

\begin{abstract}
Asynchronous failure detector-based set agreement algorithms proposed so far assume that all the processes participate in the algorithm. This means that (at least) the processes that do not crash propose a value and consequently execute the algorithm. It follows that these algorithms can block forever (preventing the correct processes from terminating) when there are correct processes that do not participate in the algorithm. This paper investigates the wait-free set agreement problem, i.e., the case where the correct participating processes have to decide a value whatever the behavior of the other processes (i.e., the processes that crash and the processes that are correct but do not participate in the algorithm). The paper presents a wait-free set agreement algorithm. This algorithm is based on a leader failure detector class that takes into account the notion of participating processes. Interestingly, this algorithm enjoys a first class property, namely, design simplicity.
\end{abstract}

Key-words: Asynchronous algorithm, Asynchronous system, Atomic register, Consensus, Leader oracle, Participating process, Set agreement, Shared object, Wait-free algorithm.

* IRISA, Université de Rennes 1, Campus de Beaulieu, 35042 Rennes Cedex, France, raynal@irisa.fr

** IRISA, Université de Rennes 1, Campus de Beaulieu, 35042 Rennes Cedex, France, corentin.travers@irisa.fr

\section{$9 \%$}

Centre National de la Recherche Scientifique (UMR 6074) Université de Rennes 1 - Insa de Rennes
Institut National de Recherche en Informatique et en Automatique - unité de recherche de Rennes 


\section{A la recherche du plus faible détecteur de fautes pour résoudre l'accord ensembliste sans attente}

Résumé : Ce rapport propose un détecteur de faute candidat à être le plus faible détecteur de fautes pour résoudre l'accord ensembliste asynchrone et sans attente.

Mots clés : Algorithme asynchrone, Registre atomique, Consensus, Oracle leader, Processus participant, Accord ensembliste, Object partagé, algorithme sans attente. 


\section{Introduction}

The consensus problem Consensus is a fundamental fault-tolerant distributed computing problem. As soon as processes cooperate, they have to agree in one way or another. This is exactly what the consensus problem captures: it allows a set of processes to agree on a critical data (called value, decision, state, etc.). Consensus can be informally defined as follows. Each process proposes a value, and a process that is not faulty has to decide a value (termination), such that there is a single decided value (agreement) ${ }^{1}$, and that value is a proposed value (validity).

It is well-known that the consensus problem cannot be solved in asynchronous systems prone to even a single process crash, be these systems read/write shared memory systems [13], or message-passing systems [5]. So, one way to circumvent this impossibility is to enrich the asynchronous system with additional objects that are strong enough to allow solving consensus.

Shared memory systems equipped with objects defined with a sequential specification and more powerful than traditional atomic read/write registers have been investigated. This line of research has produced the notion of consensus number that can be associated with each object type defined by a sequential specification [9]. The consensus number of a type is the maximum number of processes for which objects of that type (together with atomic registers) allows solving consensus. For example, the objects provided with a Test\&Set() operation have consensus number 2, while the objects provided with a Compare\&Swap () operation have consensus number $+\infty$. The consensus number notion has allowed to establish a hierarchy among the objects (with a sequential specification) according to the synchronization power of the operations they provide to the processes [9].

Another research direction has been the investigation of objects that provide processes with information on failures, namely, the objects called failure detectors [2]. A failure detector class ${ }^{2}$ is defined by abstract properties that state which information on failure is provided to the processes. According to the quality of that information, several classes can be defined. Differently from an atomic register or a Compare\&Swap object, a failure detector has no sequential specification.

As far as one is interested in solving the consensus problem in an asynchronous system prone to process crashes, it has been shown that $\Omega$ is the weakest failure detector class that allows solving consensus in such a context [3]. "Weakest" means that any failure detector that allows solving consensus provides information on failures that allows building a failure detector of the class $\Omega$.

A failure detector of the class $\Omega$ provides the processes with a primitive, denoted leader () , that returns a process identity each time it is called, and eventually always returns the same identity that is the id of a correct process, i.e., a process that does not crash when we consider crash failures. (Examples of message-passing $\Omega$-based consensus algorithms can be found in $[7,12,16]$. These algorithms assume a majority of correct processes, which is a necessary requirement for $\Omega$-based message-passing consensus algorithms.)

The set agreement problem The $k$-set agreement problem [4] generalizes the consensus problem: it weakens the constraint on the number of decided values by permitting up to $k$ different values to be decided (consensus is 1 -set agreement). While $k$-set agreement can be easily solved in asynchronous systems where the number $t$ of processes that crash is $<k$ (each of a set of $k$ predetermined processes broadcasts its value, and a process decides the first value it receives), this problem has no solution when $k \geq t[1,11,19]$. The failure detector approach to solve the $k$-set agreement problem in message-passing systems has been investigated in $[10,14,15,20]$.

While (as indicated before) it has been established that $\Omega$ is the weakest failure detector class for solving consensus [3], let us remind that finding the weakest failure failure detector class for solving $k$-set agreement for $k>1$ is still an open problem.

The main question Failure detector-based consensus algorithms implicitly consider that all the processes participate in the consensus algorithm, namely, any process that does not crash is implicitly assumed to propose a value and execute the algorithm. This is also an implicit assumption in the statement that $\Omega$ is the weakest failure detector to

\footnotetext{
${ }^{1}$ We consider here the uniform version of the consensus problem. A faulty process that decides has to decide the same value as the non-faulty processes.

${ }^{2}$ We employ the words "failure detector class" instead of "failure detector type", as it is the word traditionally used in the literature devoted to failure detectors.
} 
solve the consensus problem [3]. Basically, an $\Omega$-based consensus algorithm uses the eventual leader to eventually impose the same value to all the processes. As the algorithm does not know when the leader is elected, its main work consists in guaranteeing that no two different values can be decided before the eventual leader is elected. The algorithm uses the eventual leader to help decide all the processes that do not crash.

The previous observation raises the following question: What does happen if the process that is the eventual leader does not participate in the consensus algorithm? It appears that the algorithm can then block forever, and consequently the termination property can no longer be guaranteed.

So, a fundamental question is the following: What is the weakest failure detector to solve the consensus (or, more generally, the $k$-set agreement) problem when only a subset of the correct processes (not known a priori) propose a value and participate in the agreement problem? This question can be reformulated as follows: What are the weakest failure detector classes to wait-free solve the consensus and the $k$-set agreement problems? Wait-free means here that a process that proposes a value and does not crash has to decide, whatever the behavior of the other processes (they can participate or not, and be correct or not). The previous observation on $\Omega$ shows that a failure detector of that class cannot be the weakest to wait-free solve the consensus problem.

Content of the paper Answering the previous question requires to investigate new failure detector classes and show that one of them allows solving $k$-set agreement (sufficiency part) while being the weakest (necessity part). This paper addresses the sufficiency part. (On the necessity side, although we don't have yet formal results, we currently are inclined towards thinking that the failure detector class $\Omega_{*}^{k}$-see below- is the weakest failure detector class for wait-free solving $k$-set agreement.)

More precisely, the paper presents a failure detector-based algorithm for shared memory systems that wait-free solves the $k$-set agreement problem whatever the number $p$ of participating processes, and their behavior, in a set of $n$ processes $^{3}$. This algorithm assumes that, in addition to single-writer/multi-readers atomic registers, the shared memory provides the processes with a failure detector object of a class that we denote $\Omega_{*}^{k}$. That class is an extension of the failure detector class introduced in [18], and the failure detector classes recently introduced in [6] and [17].

The failure detector class $\Omega^{k}$ introduced in [18] extends the classical $\Omega$ class [3] by allowing a set of up to $k$ leaders to be returned by each invocation of the leader () primitive $\left(\Omega^{1}\right.$ boils down to $\Omega$ ). The set of $k$ leaders that is eventually returned forever includes at least one correct process. The aim of the class $\Omega *$ introduced in [6] is to boost obstruction-free algorithms into non-blocking algorithms. That paper also shows that this failure detector class is the weakest for such a boosting. The failure detector class introduced in [17] extends $\Omega^{k}$ by explicitly referring to the notion of participating processes. It has been introduced to circumvent the $2 p-1$ lower bound of the renaming problem [11] (where $p$ is the number of participating processes). Using such a failure detector, the proposed renaming algorithm provides the processes with a renaming space whose size is reduced from $2 p-1$ to $2 p-\left\lceil\frac{p}{k}\right\rceil$ (where the value $k$ comes from " $k$ "-set agreement).

Roadmap The paper is made up of 6 sections. Section 2 presents the computation model. Section 3 introduces the failure detector class $\Omega_{*}^{k}$. Then, Section 4 presents the $\Omega_{*}^{k}$-based $k$-set algorithm. This algorithm uses an underlying object denoted $K A$. So, Section 5 presents an algorithm constructing a $K A$ object from atomic read/write registers. Finally, Section 6 concludes the paper.

\section{Asynchronous system model}

\subsection{Process and communication model}

Process model The system consists of $n$ sequential processes that we denote $p_{1}, \ldots, p_{n}$. A process can crash. Given an execution, a process that crashes is said to be faulty, otherwise it is correct in that execution. Each process progresses at its own speed, which means that the system is asynchronous. In the following, Correct denoted the set of processes that are correct in the run that is considered.

\footnotetext{
${ }^{3}$ Let us remind that all the algorithms based on an object $O$ with consensus number $n$ allows solving consensus whatever the number $(p \leq n)$ and the behavior of the participating processes, i.e., they are wait-free consensus algorithms. (Such an object $O$ has always a sequential specification.) In some sense, this paper looks for a failure detector class that, while being as weak as possible, is as strong as the object $O$, i.e., a class that allows designing wait-free failure detector-based set agreement algorithms. (Failure detectors cannot be defined from a sequential specification.)
} 
Coordination model The processes cooperate and communicate through two types of reliable objects: two arrays of single-writer/multi-reader atomic registers and a shared object that we call $K A$ (as shown in Section 5, such an object can be built from single-writer/multi-reader atomic registers). The processes are also provided with a failure detector object of the class $\Omega_{*}^{k}$ (see below).

Identifiers with upper case letters are used to denote shared objects. Lower case letters are used to denote local variables; in that case the process index appears as a subscript. As an example, $\operatorname{part}_{i}[j]$ denotes the $j$ th entry of a local array of the process $p_{i}$, while $P A R T[j]$ denotes the $j$ th entry of the shared array $P A R T$.

\subsection{The $K A$ object}

The $K A$ object is a variant of a round-based object introduced in [8] to capture the safety properties of Paxos-like consensus algorithms $[8,12]$. This object provides the processes with an operation denoted alpha propose ${ }_{k}()$. That operation has two input parameters: the value $\left(v_{i}\right)$ proposed by the invoking process $p_{i}$, and a round number $\left(r_{i}\right)$. The round numbers play the role of a logical time and allows identifying the invocations. The $K A$ object assumes that no two processes use the same round numbers, and successive invocations by the same process use increasing round numbers. Given a $K A$ object, the invocations alpha $\operatorname{propose}_{k}()$ satisfy the following properties ( $\perp$ is a default value that cannot be proposed by a process):

- Termination (wait-free): an invocation of alpha propose $_{k}()$ by a correct process always terminates (whatever the behavior of the other processes).

- Validity: the value returned by any invocation alpha $\operatorname{propose}_{k}()$ is a proposed value or $\perp$.

- Agreement: At most $k$ different non- $\perp$ values can be returned by the whole set of al pha propose ${ }_{k}()$ invocations.

- Convergence: If there is a time after which the operation alpha propose ${ }_{k}()$ is invoked infinitely often, and these invocations are issued by an (unknown but fixed) set of at most $k$ processes, there is then a time after which none of these invocations returns $\perp$.

A $K A$ object can store up to $k$ non- $\perp$ different values. A process invokes it with a value to store and obtains a value in return. If it is permanently accessed concurrently by more than $k$ processes, the $K A$ object might store anything. If there is a period during which it is accessed concurrently by at most $k^{\prime} \leq k$ processes, it stores forever the corresponding $k^{\prime}$ proposed values.

\section{The failure detector class $\Omega_{*}^{k}$}

\subsection{Definition}

A failure detector of the class $\Omega_{*}^{k}$ provides the processes with an operation denoted leader(). (As indicated in the introduction, this definition is inspired by the leader failure detector classes introduced in $[6,17,18]$.) When a process $p_{i}$ invokes that operation, it provides it with an input parameter, namely a set $X$ of processes, and obtains a set of process identities as a result ${ }^{4}$.

The semantics of $\Omega_{*}^{k}$ is based on a notion of time, whose domain is the set of integers. It is important to notice that this notion of time is not accessible to the processes. An invocation of leader $(X)$ by a process $p_{i}$ is meaningful if $i \in X$. If $i \notin X$, it is meaningless. The primitive leader() is defined by the following properties:

- Termination (wait-free): Any invocation of leader() by a correct process always terminates (whatever the behavior of the other processes).

- Triviality: A meaningless invocation returns any set of processes.

- Eventual multi-leadership for each input set $X$ : For any $X \subseteq \Pi$, such that $X \cap$ Correct $\neq \emptyset$, there is a time $\tau_{X}$ such that, $\forall \tau \geq \tau_{X}$, all the meaningful leader $(X)$ invocations (that terminate) return the same set $L_{X}$ and this set is such that:

\footnotetext{
${ }^{4}$ The definition of $\Omega_{*}^{k}$ is not expressed in the framework introduced by Chandra and Toueg to define failure detector classes [2]. More precisely, in their framework, the failure detector operation that a process can issue has no input parameter. It would be possible to express $\$ \mathbf{k}$ in their framework. We don't do it in order to make the presentation simpler.
}

PI ${ }^{\circ} 1811$ 


$$
\begin{aligned}
& \text { - }\left|L_{X}\right| \leq k . \\
& \text { - } L_{X} \cap X \cap \text { Correct } \neq \emptyset .
\end{aligned}
$$

The intuition that underlies this definition is the following. The set $X$ passed as input parameter by the invoking process $p_{i}$ is the set of all the processes that $p_{i}$ considers as being currently participating in the computation. (This also motivates the notion of meaningful and meaningless invocations: an invoking process is trivially participating).

Given a set $X$ of participating processes that invoke leader $(X)$, the eventual multi-leadership property states that there is a time after which these processes obtain the same set $L_{X}$ of at most $k$ leaders, and at least one of them is a correct process of $X$. Let us observe that the (at most $k-1$ ) other processes of $L_{X}$ can be any subset of processes (correct or not, participating or not).

It is important to notice that the time $\tau_{X}$ from which this property occurs is not known by the processes. Moreover, before that time, there is an anarchy period during which each process, as far as its leader $(X)$ invocations are concerned, can obtain different sets of any number of leaders. Let us also observe that if a process $p_{i}$ issues two meaningful invocations leader $(X 1)$ and leader $(X 2)$ with $X 1 \neq X 2$, there is no relation linking $L_{X 1}$ and $L_{X 2}$, whatever the values of $X 1$ and $X 2$ (e.g., the fact that $X 1 \subset X 2$ imposes no particular constraint on $L_{X 1}$ and $L_{X 2}$ ).

Let us consider an execution in which all the invocations leader $(X)$ are such that $X=\Pi$ (the whole set of processes are always considered as participating). In that case, $\Omega_{*}^{k}$ boils down to the failure detector class denoted $\Omega^{k}$ introduced in [18]. If additionally, $k=1$, we obtain the classical leader failure detector $\Omega$ introduced in [3]. When $k=1, \Omega_{*}^{k}$ boils down to the failure detector class introduced in [6]. It is shown in [6] that $\Omega$ is weaker than $\Omega_{*}^{1}$ that in turn is weaker than $\diamond \mathcal{P}$ (the class of eventually perfect failure detectors: after some finite but unknown time, these failure detectors suspect all the crashed processes and only them [2]).

\subsection{The family $\left\{\Omega_{*}^{k}\right\}_{1 \leq k \leq n}$}

It follows from the definition of $\Omega_{*}^{k}$, that the failure detector class family $\left\{\Omega_{*}^{k}\right\}_{1 \leq k \leq n}$ is such that $\Omega_{*}^{k} \subset \Omega_{*}^{k+1}$.

Moreover, as just indicated, when all the leader $(X)$ invocations are such that $X=\Pi, \Omega_{*}^{k}$ boils down $\Omega^{k}$ (as defined in [18]), from which it follows that we have $\Omega^{k} \subseteq \Omega_{*}^{k}$. More generally, the failure detector classes $\Omega^{k}$ and $\Omega_{*}^{k}$ are related as indicated in Figure 1 where $A \rightarrow B$ means that any failure detector of the class $A$ can be used to build a failure detector of the class $B$, and $A \cdots>B$ means that it is not possible to build a failure detector of the class $B$ from a failure detector of the class $A$.

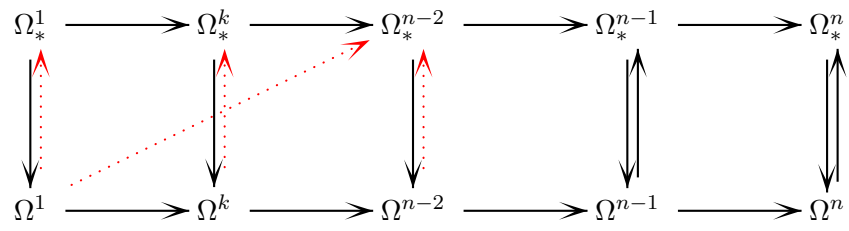

Figure 1: Wait-free (ir)reducibility results between the families $\left(\Omega_{*}^{x}\right)_{1 \leq x \leq n}$ and $\left(\Omega^{y}\right)_{1 \leq y \leq n}$

- The fact that $\Omega^{k} \subseteq \Omega_{*}^{k}$ (top-down plain arrows) follows from the definitions of the failure detector classes.

- The fact that $\Omega^{n}$ and $\Omega_{*}^{n}$ are the same class (top-down and bottom-up arrows at the right) follows directly from their definitions.

- The fact that $\forall k: 1 \leq k<n-1, \forall k^{\prime}: 1 \leq k^{\prime} \leq n$, it is not possible to build a failure detector of the class $\Omega_{*}^{k}$ from a failure detector of the class $\Omega^{k^{\prime}}$ (dotted arrows) is established in Theorem 1 .

- The fact that it is possible to construct a failure detector of the class $\Omega_{*}^{n-1}$ from any failure detector of the class $\Omega^{n-1}$ is established in Theorem 2 .

Theorem $1 \forall k: 1 \leq k<n-1, \forall k^{\prime}: 1 \leq k^{\prime} \leq n$, it is not possible to build a failure detector of the class $\Omega_{*}^{k}$ from a failure detector of the class $\Omega^{k^{\prime}}$. 
Proof To prove the theorem, it suffices to show that it is not possible to build a failure detector of the class $\Omega_{*}^{n-2}$ (the weakest class in the family $\left.\left(\Omega_{*}^{k}\right)_{1 \leq k \leq n-2}\right)$ from a failure detector of the class $\Omega^{1}$ (the strongest class in the family $\left.\left(\Omega^{k}\right)_{1 \leq k \leq n}\right)$. The proof is by contradiction. Let us assume that there is an algorithm $\mathcal{A}$ that builds a failure detector of the class $\Omega_{*}^{n-2}$ from a failure detector of the class $\Omega^{1}\left(^{5}\right)$. We construct an infinite run in which at least one of the failure detectors $\Omega^{1}$ or $\Omega_{*}^{n-2}$ fails to meet its specification. The construction uses the following claim. Let LEADER() denote the leader primitive of $\Omega^{1}$. Recall that leader $(X)$ is the leader primitive of $\Omega_{*}^{n-2}$.

Claim $C$. Let $R$ be an arbitrary run in which each process is correct. Moreover, in run $R$, each process $p_{i}$ periodically invokes leader $(X)$ for each $X$ such that $i \in X \wedge|X|=n-1$. Let $\tau$ be a time at which the leadership properties of both $\Omega^{1}$ and $\Omega_{*}^{n-2}$ are satisfied (i.e., all the invocations of LEADER() return the same process id, and, for any set $X$, all the invocations leader $(X)$ return the same set). We claim that there is a run $R_{1}$ of the algorithm $\mathcal{A}$ such that

- $R_{1}$ is indistinguishable from $R$ up to time $\tau$.

- In $R_{1}$, there is a process $p_{x}$ and a time $\tau_{1}>\tau$ such that (1) the outputs of $\Omega^{1}$ at $\tau$ and $\tau_{1}$ are different, or (2) the outputs of $\Omega_{*}^{n-2}$ (for some set $X$ ) at $\tau$ and $\tau_{1}$ are different.

- No process crashes in $R_{1}$.

Proof of the claim. By the claim assumption, the leadership properties of both $\Omega^{1}$ and $\Omega_{*}^{n-2}$ are satisfied at time $\tau$ in $R$. In particular, we have:

1. $\Omega^{1}$ outputs at each process the same leader identity $\ell$.

2. Let $X=\Pi-\{\ell\}$. At time $\tau, \exists L$ such that $|L| \leq n-2$, and, for each process $p_{i} \in X$, $\operatorname{leader}(X)=L$. Let $L^{\prime}=L \cap X$. We have $\left|L^{\prime}\right| \leq n-2$. As $|X|=n-1$, there is a process $p_{x}$ such that $x \in X-L^{\prime}$. Moreover, $x \neq \ell$ (because $X=\Pi-\{\ell\}$ ).

We show that, from the previous observations, we can build a run $R_{1}$, identical to $R$ up to time $\tau$, such that there exists a time $\tau^{\prime}>\tau$ at which we have:

- $\Omega^{1}$ outputs at some process a leader $\ell_{1} \neq \ell$ or,

- At process $p_{x}$, leader $(X) \neq L$.

Let us consider the run $R^{\prime}$ defined as follows. $R^{\prime}$ is the same as $R$ up to time $\tau$. At time $\tau+1$, every process in $L^{\prime}$ crashes. Moreover, from $\tau$, all the invocations LEADER() of $\Omega^{1}$ output $\ell$ in $R^{\prime}$. Due to the eventual multi-leadership property of $\Omega_{*}^{n-2}$, there is a time $\tau_{1}>\tau+1$ such that the invocations of leader $(X)$ at process $p_{x}$ return $L_{1} \neq L$. This is because the set that is returned (namely, $L_{1}$ ) has to be such that $L_{1} \cap X \cap$ Correct $\neq \emptyset$, and, as the processes of $L^{\prime}$ have crashed, we have $L \cap X \cap$ Correct $=\emptyset$ (recall that $\left.L^{\prime}=L \cap X\right)$.

Let us now consider the run $R_{1}$ identical to $R$ up to time $\tau+1$. During the interval $\left[\tau+1, \tau_{1}\right]$ the processes in $L^{\prime}$ do not take any step as far the algorithm $\mathcal{A}$ is concerned. The other processes behave exactly as in $R^{\prime}$. If $\Omega^{1}$ outputs $\ell^{\prime} \neq \ell$ at some process $p_{y}$, the claim follows. Otherwise, let us observe that, for any process $p_{y}$ (such that $y \notin L^{\prime}$ ), $R_{1}$ cannot be distinguished from $R^{\prime}$. In particular, the algorithm $\mathcal{A}$ outputs, at process $p_{x}, L_{1} \neq L$ at time $\tau_{1}$ when $p_{x}$ issues leader $(X)$. End of the proof of the claim.

Let us consider an arbitrary run $R_{0}$ in which each process is correct. There is a time $\tau_{1}(1)$ at which the leadership properties of both failure detectors $\Omega^{1}$ and $\Omega_{*}^{n-2}$ are satisfied, and (2) each process has taken at least one step. By Claim $C$, we can build a run $R_{1}$ identical to run $R$ up to time $\tau_{1}$ and such that the output of $\Omega^{1}$ or $\Omega_{*}^{n-2}$ (for some input parameter $X$ ) at some process has changed at time $\tau_{1}^{\prime}>\tau_{1}$.

In run $R_{1}$, we can find a time $\tau_{2}>\tau_{1}^{\prime}$ such that each process has taken at least one step between $\tau_{1}^{\prime}$ and $\tau_{2}$ and the leadership properties of $\Omega_{1}$ and $\Omega_{*}^{n-2}$ are satisfied at time $\tau_{2}$. By applying Claim $C$, we can build a run $R_{2}$ identical to $R_{1}$ up to time $\tau_{2}$, etc. By iterating this process, we obtain an infinite run $R$ and an infinite sequence of increasing times $\left(\tau_{1}, \tau_{1}^{\prime}, \tau_{2}, \tau_{2}^{\prime}, \ldots\right)$ such that $\forall i>0, \exists p_{x(i)}$ such that, at $p_{x(i)}$ the output of $\Omega^{1}$ or $\Omega_{*}^{n-2}$ (for some parameter $X$ ) is not the same at time $\tau_{i}$ and $\tau_{i}^{\prime}$. Due to the eventual leadership property of $\Omega$, there is a time after which the output

\footnotetext{
${ }^{5}$ Let us recall that the output of $\Omega^{1}$ at a given process $p_{i}$ is local. This means that for the output of $\Omega^{1}$ at $p_{i}$ be known by the thread implementing the algorithm $\mathcal{A}$ at $p_{j}$, it is necessary that that output be written in the shared memory.
}

PI $n^{\circ} 1811$ 
of $\Omega^{1}$ does not change at each process. Consequently, it follows that in run $R$ algorithm $\mathcal{A}$ fails to implement $\Omega_{*}^{n-2}$.

$\square$ Theorem 1

Theorem 2 Given any failure detector of the class $\Omega^{n-1}$, it is possible to build a failure detector of the class $\Omega_{*}^{n-1}$.

Proof The proof is constructive. Let us consider any failure detector of the class $\Omega^{n-1}$, and let LEADER() be its leader primitive. Let us consider the operation leader $(X)$ defined as follows:

operation leader $(X)$ : if $X=\Pi$ then return (LEADER ()$)$ else return $(X)$ end_if.

We show that leader $(X)$ satisfies the properties of the class $\Omega_{*}^{n-1}$.

Let us first consider the case where $X=\Pi$. Due to the properties of $\Omega^{n-1}$, there is a time after which LEADER() always returns the same set $L_{X}$ such that $\left|L_{X}\right|=n-1$ and $L_{X} \cap$ Correct $\neq \emptyset$. It trivially follows that $X \cap L_{X} \cap$ Correct $\neq \emptyset$. Consequently, the eventual multi-leadership property is satisfied for the invocations leader $(\Pi)$.

Given any set $X$ such that $X \neq \Pi$, let us now consider the case of the invocations leader $(X)$. The definition of leader $(X)$ indicates that the set $L_{X}=X$ is then returned by these invocations, and we have $|X|=\left|L_{X}\right| \leq n-1$. If $X$ contains at least one correct process, we have $X \cap L_{X} \cap$ Correct $\neq \emptyset$, and the eventual multi-leadership property is satisfied for the invocations leader $(X)$. If $X$ contains no correct process, the set returned by leader $(X)$ can be arbitrary.

$\square_{\text {Theorem } 2}$

\section{$4 \Omega_{*}^{k}$-based $k$-set agreement}

\subsection{Wait-free $k$-set agreement}

The $k$-set agreement has been informally stated in the introduction. It has been defined in [4]. The parameter $k$ of the set agreement can be seen as the degree of coordination associated with the corresponding instance of the problem. The smaller $k$, the more coordination among the processes: $k=1$ means the strongest possible coordination (this is the consensus problem), while $k=n$ means no coordination at all. More precisely, in a set of $n$ processes, each of a subset of $p \geq 1$ processes proposes a value. These processes are the participating processes. The wait-free $k$-set agreement is defined by the following properties:

- Termination (wait-free): a correct process that proposes a value decides a value (whatever the behavior of the other processes).

- Agreement: no more than $k$ different values are decided.

- Validity: a decided value is a proposed value.

\subsection{Principles and description of the algorithm}

The $k$-set agreement algorithm is described in Figure 2. A process $p_{i}$ that participates in the $k$-set agreement invokes the operation kset_propose ${ }_{k}\left(v_{i}\right)$ where $v_{i}$ is the value it proposes. If it does not crash, it terminates that operation when it executes the statement return $\left(\right.$ decided $\left._{i}\right)$ (line 11) where decided $_{i}$ is the value it decides.

Shared objects The processes share three objects:

- A $K A$ object. A process $p_{i}$ accesses it by invoking $K A$.alpha_propose ${ }_{k}\left(r_{i}, v_{i}\right)$ where $r_{i}$ is a round number and the value $v_{i}$ proposed by $p_{i}$ (line 07 ). Due to the properties of the $K A$ object, the value returned by such an invocation is a proposed value or $\perp$.

- An array of atomic single-writer/multi-reader boolean registers, $P A R T[1 . . n]$. The register PART $[i]$ can be updated only by $p_{i}$; it can read by all the processes. Each entry $P A R T[i]$ is initialized to false. PART $[i]$ is switched to true to indicate that $p_{i}$ is now participating in the $k$-set agreement (line 01$) . P A R T[i]$ is updated at most once. 
- An array of atomic single-writer/multi-reader registers denoted $D E C[1 . . n] . D E C[i]$ can be written only by $p_{i}$. It can read by all the processes. Each entry $D E C[i]$ is initialized to $\perp$ (a value that cannot be proposed by the processes). When it is updated to a non- $\perp$ value $v$, that value $v$ can be decided by any process. It is updated (to such a value $v$ or $\perp$ ) each time $p_{i}$ invokes $K A$.alpha_propose $k_{k}\left(r_{i}, v_{i}\right)$ to store the value returned by that invocation (line 07).

The algorithm The behavior of a process is pretty simple. As in Paxos, it decouples the safety part from the waitfree/termination part. The safety is ensured thanks to the $K A$ object, while the liveness rests on $\Omega_{*}^{k}$.

After it has registered its participation (line 01), a process $p_{i}$ executes a while loop (lines 03-09) until it finds a non- $\perp$ entry in the $D E C[1 . . n]$ array. When this occurs, $p_{i}$ decides such a value (lines 03 and 10-11).

Each time it executes the while loop, $p_{i}$ first computes its local view (denoted part $_{i}$ ) of the set of the processes it perceives as being the participating processes (line 04). It then uses this participating set to invoke Leader ${ }_{i}()$ (line 05). If it does not belong to the set returned by Leader ${ }_{i}\left(\right.$ part $\left._{i}\right), p_{i}$ continues looping. Otherwise (it then belongs to set of leaders), $p_{i}$ invokes the $K A$ object (line 07 ) to try to obtain a non- $\perp$ value from that object. The local variable $r_{i}$ is used by $p_{i}$ to define the round number it uses when it invokes the $K A$ object. It is easy to see from the management of $r_{i}$ at line 02 and line 06 that each process uses increasing round numbers, and that no two processes use the same round numbers (a necessary requirement for using the $K A$ object). The properties of $K A$ ensure that no more than $k$ values are decided, while the properties of $\Omega_{*}^{k}$ ensure that all the correct participating processes do terminate.

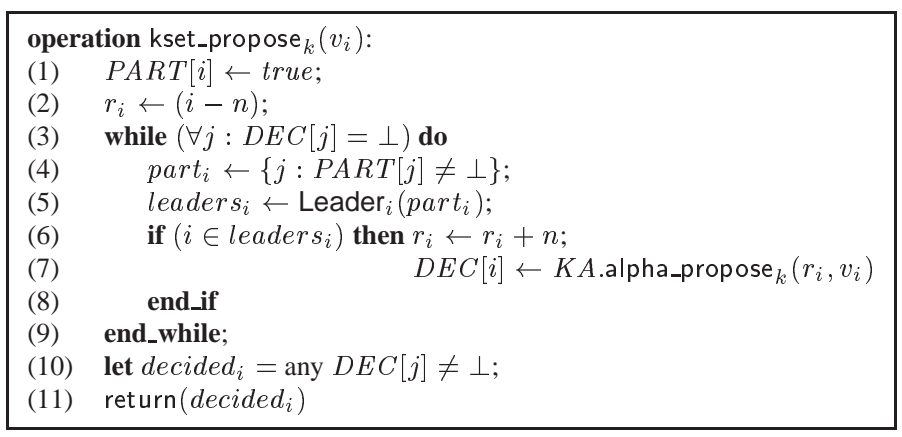

Figure 2: An $\Omega_{*}^{k}$-based $k$-set agreement algorithm (code for $p_{i}$ )

\subsection{Proof of the algorithm}

Theorem 3 The algorithm described in Figure 2 wait-free solves the $k$-set agreement problem whatever the number p of participating processes in a set of n processes (this number p being a priori unknown).

\section{Proof}

Validity The validity property follows from the following observations:

- The value $\perp$ cannot decided (lines 03 and 10).

- The $D E C[1 . . n]$ array can contain only $\perp$ or values that have been proposed to the $K A$ object (line 07 ).

- Any value $v_{i}$ proposed to the $K A$ object is a value proposed to the $k$-set agreement.

Agreement The agreement property follows directly from the agreement property of the $K A$ object (that states that at most $k$ non- $\perp$ values can be returned from that object). 
Termination (wait-free) If an entry of $D E C[1 . . n]$ is eventually set to a non- $\perp$ value, it follows from the test of line 03 that any correct participating process terminates. So, let us assume by contradiction that no entry of $D E C[1$..n] is ever set to a non- $\perp$ value. Let us first observe that all the leader() invocations issued by the processes are meaningful.

If no correct participating process decides, there is a time $\tau_{0}$ after which we have the following:

- All the participating processes have entered the algorithm, and consequently the array PART[1..n] determines the whole set of participating processes. Let $X$ be this set of processes.

- all the leader() invocations have $X$ as input parameter.

It follows from the eventual multi-leadership property associated with $X$, that there is a time $\tau_{X} \geq \tau_{0}$ such that, for all the times $\tau \geq \tau_{X}$, all the invocations of leader $(X)$ return the same set $L_{X}$ of at most $k$ processes, and this set includes at least one correct participating process.

As no process decides (assumption) and each alpha propose ${ }_{k}()$ invocation issued by a correct process returns (termination property of the $K A$ object), the correct participating processes of the set $X$ execute $K A$.alpha propose ${ }_{k}()$ infinitely often (lines 06-07). It then follows from the convergence property of the $K A$ object that these processes obtain non- $\perp$ values, and deposit these values in the array $D E C[1 . . n]$. A contradiction.

$\square$ Theorem 3

\section{Building a $K A$ object from registers}

This section presents an implementation of a $K A$ object from single-writer/multi-readers atomic registers. As already indicated, this algorithm is inspired from Paxos-like algorithms $[8,12]$.

\subsection{Implementing $K A$}

An algorithm constructing a $K A$ object is described in Figure 3. It uses an array of single-writer/multi-reader atomic registers $R E G$. As previously, $R E G[i]$ can be written only by $p_{i}$. A register $R E G[i]$ is made up of three fields $R E G[i] . l r e, R E G[i] . l r w w$ and $R E G[i]$.val whose meaning is the following $(R E G[i]$ is initialized to $<0,0, \perp>)$ :

- $R E G[i] . l r e$ stores the number of the last round entered by $p_{i}$. It can be seen as the logical date of the last invocation issued by $p_{i}$.

- $R E G[i] . l r w w$ and $R E G[i] . v a l$ constitute a pair of related values: $R E G[i] . l r w w$ stores the number of the $l$ ast round with a write of a value in the field $R E G[i] . v a l$. So, $R E G[i] . l r w w$ is the logical date of the last write in $R E G[i] . v a l$.

(To simplify the writing of the algorithm, we consider that each field of a register can be written separately. This poses no problem as each register is single writer. A writer can consequently keep a copy of the last value it has written in each register field and rewrite it when that value is not modified.)

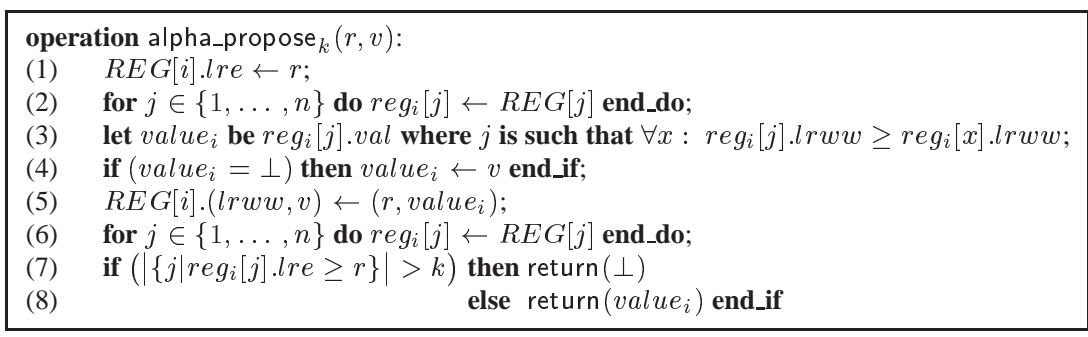

Figure 3: A $K A$ object algorithm (code for $\left.p_{i}\right)$

The principle that underlies the algorithm is very simple: it consists in using a logical time frame (represented here by the round numbers) to timestamp the invocations, and answering $\perp$ when the timestamp of the corresponding invocation does not lie within the $k$ highest dates (registered in $R E G[1 . . n] . l r e$ ). To that end, the algorithm proceeds as follows: 
- Step 1 (lines 01-02): Access the shared registers.

- When a process $p_{i}$ invokes alpha_propose ${ }_{k}(r, v)$, it first informs the other processes that the $K A$ object has attained (at least) the date $r$ (line 01). Then $p_{i}$ reads all the registers in any order (line 02) to know the last values (if any) written by the other processes.

- Step 2 (lines 03-05): Determination and writing of a value.

Then, $p_{i}$ determines a value. In order not to violate the agreement property, it selects the last value ("last" according to the round numbers/logical dates) that has been deposited in a register $R E G[j]$. If there is no such value it considers its own value $v$. After this determination, $p_{i}$ writes in $R E G[i]$ the value it has determined, together with its round number (line 05 .

- Step 3 (lines 06-08): Commit or abort.

- $p_{i}$ reads again the shared registers to know the progress of the other processes (measured by their round numbers), line 07. If it discovers it is "late", $p_{i}$ aborts returning $\perp$. (Let us observe that this preserves the agreement property.) "To be late" means that the current date $r$ of $p_{i}$ does not lie within the window defined by the $k$ highest dates (round numbers) currently entered by the processes (these round numbers/dates are registered in the field lre of each entry of the array $R E G[1 . . n])$.

- Otherwise, $p_{i}$ is not late. It then returns ("commits") value ${ }_{i}$ (line 08). Let us observe that, as the notion of "being late" is defined with respect to a window of $k$ dates (round numbers), it is possible that up to $k$ processes are not late and return concurrently up to $k$ distinct non- $\perp$ values.

It directly follows from the code that the algorithm is wait-free. Moreover, in order to expedite the alpha propose ${ }_{k}()$ operation, it is possible to insert the statement

$$
\text { if }\left(\left|\left\{j \mid r e g_{i}[j] . l r e \geq r\right\}\right|>k\right) \text { then return }(\perp) \text { end_if }
$$

between the line 02 and the line 03. This allows the invoking process to return $\perp$ when, just after entering the alpha_propose ${ }_{k}()$ operation, it discovers it is late.

\subsection{Proof of the $K A$ object}

Theorem 4 The algorithm described in Figure 3 wait-free implements a KA object.

Proof

Termination (wait-free) This property follows directly from the code of the algorithm (the only loops are for loops that trivially terminate when the invoking process is correct).

Validity Let us observe that if a value $v$ is written in $R E G[i] . v a l$, that value has been previously passed as a parameter in an alpha_propose ${ }_{k}()$ invocation. The validity property follows from this observation and the fact that only $\perp$

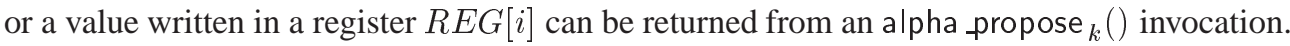

Convergence Let $\tau$ be a time after which there is a set of $k^{\prime} \leq k$ processes such that each of them invokes alpha_propose ${ }_{k}()$ infinitely often. This means that, from $\tau$, the values of $n-k^{\prime}$ registers $R E G[x]$ are no longer modified. Consequently, as the $k^{\prime}$ processes $p_{j}$ repeatedly invoke alpha propose ${ }_{k}()$, there is a time $\tau^{\prime} \geq \tau$ after which each $R E G[j]$.lre becomes greater than any $R E G[x]$.lre that is no longer modified. There is consequently a time $\tau^{\prime \prime} \geq \tau^{\prime}$ after which the $k^{\prime}$ processes are such that their registers $R E G[j]$.lre contain forever the $k$ greatest timestamp values. It follows from the test done at line 07 that, after $\tau^{\prime \prime}$, no alpha_propose ${ }_{k}()$ invocation by one of these $k^{\prime}$ processes can be aborted. Consequently, each of them returns a non- $\perp$ value at line 08 .

Agreement If all invocations returns $\perp$, the agreement property is trivially satisfied. So, let us consider an execution in which at least one alpha_propose ${ }_{k}()$ invocation returns a non- $\perp$ value. To prove the agreement property we show that: 
- Before the first non- $\perp$ value is returned by an invocation, there is a time at which the algorithm has determined a set $V$ of at least one and at most $k$ non- $\perp$ values $^{6}$.

- Any value $v \neq \perp$ returned by an invocation is a value of $V$.

To simplify the reasoning, and without loss of generality, we assume that a process that repeatedly invokes alpha_propose ${ }_{k}()$, stops invoking that operation as soon as it returns a non- $\perp$ value at line 08 .

1. Invariants. $\forall j \in\{1, \ldots, n\}$ :

- $R E G[j] . l r e$ is increasing (assumption on the successive round numbers used by $p_{j}$ ).

- $R E G[j] . l r w w \leq R E G[j]$.lre (because $p_{j}$ executes line 05 after line 01).

2. Among all the invocations that execute the test of line 07 , let $\mathcal{I}$ be the subset of invocations for which the predicate $\mid\left\{j \mid \mathrm{reg}_{i}[j]\right.$.lre $\left.\geq r\right\} \mid \leq k$ is true. (This means that any invocation of $\mathcal{I}$ either returns a non- $\perp$ value -at line 08-, or crashes after it has evaluated the predicate at line 07, and before it executes line 08.) Among the invocations of $\mathcal{I}$, let $I$ be the invocation with the smallest round number. Let $p_{j_{1}}$ be the process that invoked $I$ and $r$ the corresponding round number.

3. Time instants (see Figure 4).

- Let $\tau$ be the time at which $I$ executes line 05 (statement $R E G\left[j_{1}\right] \leftarrow<r, r, v>$ ).

- Let $\tau^{\prime}$ be the time just after $I$ has finished reading the array $R E G[1 . . n]$. Without loss of generality, we consider that this is the time at which $I$ locally evaluates the predicate of line 07 .

- Let $\tau[j]$ be the time at which $I \operatorname{reads} R E G[j]$ at line 06 . We have $\tau<\tau[j]<\tau^{\prime}$.

\begin{tabular}{ccc}
$\tau$ & $\tau[j]$ & $\tau^{\prime}$ \\
\hdashline$\vdots$ & $\vdots$ \\
$R E G\left[j_{1}\right] \leftarrow<r, r, v>$ & $r e g_{j_{1}}[j] \leftarrow R E G[j]$ & $\left|\left\{j \mid r e g_{j_{1}}[j] \geq r\right\}\right| \leq k$ is satisfied \\
line 05 & line 06 & line 07
\end{tabular}

Figure 4: Time instants with respect to accesses to the registers $R E G[1 . . n]$

4. From $\tau[j]<\tau^{\prime}$, the fact that predicate $\mid\left\{j \mid r e g_{j_{1}}[j]\right.$.lre $\left.\geq r\right\} \mid \leq k$ is true at $\tau^{\prime}$, and the monotonicity of $R E G[j]$.lre, we can conclude that a necessary requirement for the predicate $R E G[j] . l r e \geq r$ to be true at $\tau$ is that it is true at $\tau^{\prime}$.

Let $L=\left\{j_{1}, \ldots, j_{x}, \ldots, j_{\ell}\right\}$ be the set of processes $p_{j}$ such that $R E G[j] . l r e \geq r$ is true at $\tau$. As the predicate $\mid\left\{j \mid\right.$ reg $_{i}[j]$.lre $\left.\geq r\right\} \mid \leq k$ is true at $\tau^{\prime}$, we have $1 \leq \ell=|L| \leq k$.

5. From the previous item, we conclude that there are at least $n-\ell \geq n-k$ entries $j$ of the array $R E G[1 . . n]$ such that $R E G[j]$.lre $<r$ at time $\tau$. Let $\bar{L}$ denote this set of processes ( $L$ and $\bar{L}$ define a partition of the whole set of processes).

6. Let the $\tau$-time invocation of $p_{j}$ be the invocation issued by $p_{j}$ whose round number is the value of $R E G[j] . l r e$ at time $\tau$ (assuming a fictitious initial invocation if needed).

7. The $\tau$-time invocations of the processes $p_{j}$ in $L$ define a set, denoted $V$, including at most $\ell \leq k$ values, such that these values are written in $R E G[1 . . n]$ with a write timestamp (value of the field $R E G[j] . l r w w$ ) that is $\geq r$. This claim follows from the following observation.

- The $\tau$-time invocation by $p_{j_{1}}$ (namely $I$ ) writes a value and the round number $r$ in $R E G\left[j_{1}\right]$.

- Let $p_{j_{x}} \in L, p_{j_{x}} \neq p_{j_{1}}$. From the definition of $L$, it follows that the round number of the $\tau$-time invocation issued by $p_{j_{x}}$ is $R E G\left[j_{x}\right] . l r e=r^{\prime}>r$. When it executes that invocation, $p_{j_{x}}$ atomically executes $R E G\left[j_{x}\right] \leftarrow<r^{\prime}, r^{\prime}, v^{\prime}>$ (if it does not crash before executing the line 05).

\footnotetext{
${ }^{6}$ According to the terminology introduced in [2], the set $V$ defines the values that are locked. This means that from now on the set of non- $\perp$ values that can be returned is fixed forever: no value outside $V$ can ever be returned.
} 
- It is possible that, on one side, no process in $L$ crashes before executing line 05 , and, on another side, all the values that are written are different. It consequently follows that up to $\ell \leq k$ different values (with a write timestamp $l r w w \geq r$ ) can be written in $R E G[1 . . n]$. Hence, $V$ can contain up to $k$ values.

- Moreover, it is also possible that each process in $L$ returns at line 08 the value it has selected at line 05 (this depends on the value of the predicate evaluated at line 07). Consequently each value of $V$ can potentially be returned.

8. Given an execution, the previous item has extracted a non-empty set $V$ of at most $k$ non- $\perp$ values that can be returned. We now show that (1) from $\tau$, only values of $V$ can be written in $R E G[1 . . n]$ with a timestamp field (lrww) greater than $r$, and (2) a non- $\perp$ value returned by an invocation is necessarily a value of $V$.

(a) The $\tau$-time invocation issued by any $p_{j} \in \bar{L}$ has a round number $R E G[j]$.lre that is smaller than $R E G\left[j_{1}\right] . \operatorname{lr} e=$ $r$ (this follows from the definition of $\bar{L}$ ). As by definition, $r$ is the smallest round number during which a process finds true the predicate of line 07 , it follows that any $p_{j} \in \bar{L}$ needs to issue an invocation with a round number greater than $r$ to have a chance to return a non- $\perp$ value.

(b) Let $\mathcal{I}^{\prime}$ be the set of all the invocations that have a round number greater than $r$. They are issued by the processes of $\bar{L}$ or the processes of $L$ whose $\tau$-time invocation has returned $\perp$ at line 07 . Let us observe that any invocation of $\mathcal{I}^{\prime}$ starts after $\tau$.

Let $I^{\prime}$ be the first invocation of $\mathcal{I}^{\prime}$ that executes 05 . $I^{\prime}$ (issued by some process $p_{j}$ ) selects (at line 03 ) a

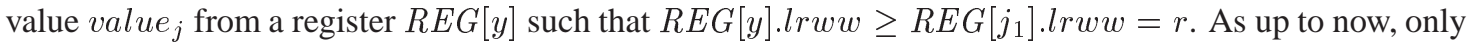
processes of $L$ have written values in $R E G[1 . . n]$ with a write timestamp (lrww) $\geq r$, it follows that $I^{\prime}$ selects a value from $V^{7}$. Consequently, this invocation does not add a new value to $V$.

Let $I^{\prime \prime}$ be the invocation of $\mathcal{I}^{\prime}$ that is the second to execute line 05 . The same reasoning (including now $I^{\prime}$ ) applies. Etc. It follows from this induction that a value written at line 05 by an invocation of $\mathcal{I}^{\prime}$ is a value of $V$, which proves that only values of $V$ can be written in the array $R E G[1 . . n]$ with a write timestamp greater than $r$.

(c) Finally, an invocation that returns a value at line 08, returns the value it has written at line 05. Due to the definition of $r$, its round number $r^{\prime}$ is $\geq r$. It follows that the non- $\perp$ value that is returned is a value of $V$.

\section{Conclusion}

Considering asynchronous systems equipped with a failure detector object, this paper focused on the set agreement problem when only a subset of the processes participate, namely, the wait-free set agreement problem. Wait-free means here that a correct process has to decide a value, whatever the behavior of the other processes (that can be correct or not and participate or not).

A wait-free failure detector-based $k$-set agreement algorithm has been presented. Its design principles follows the Paxos approach, decoupling the way the safety and the termination properties are guaranteed. The algorithm safety is based on an object denoted $K A$ that can be built from single-writer/multi-reader atomic registers. The liveness property is based on a leader failure detector class, denoted $\Omega_{*}^{k}$, that takes into account the participating processes. The very existence of the algorithm shows that $\Omega_{*}^{k}$ is sufficient to wait-free solve the $k$-set agreement problem. Showing that $\Omega_{*}^{k}$ is also necessary, or defining a class of weaker failure detectors solving the $k$-set agreement problem, remains one of the greatest research challenges for the fault-tolerant asynchronous computing theory community.

\section{References}

[1] Borowsky E. and Gafni E., Generalized FLP Impossibility Results for $t$-Resilient Asynchronous Computations. Proc. 25th ACM Symposium on Theory of Computation (STOC'93), San Diego (CA), pp. 91-100, 1993.

\footnotetext{
${ }^{7}$ It is possible that, when $I^{\prime}$ reads the array $R E G[1 . . n]$ at line 02 , not all the values of $V$ have yet been written in that array. The important points are here that (1) at least one value of $V$ has already been written in the array (namely, $R E G[\dot{\lambda}] . v a l$ with the timestamp $R E G\left[j_{1}\right] . l r w w=r$ ), and (2) any register $R E G[x]$ that currently contains a value not in $V$, is such that $R E G[x] . \operatorname{lr} w w<r$.
} 
[2] Chandra T. and Toueg S., Unreliable Failure Detectors for Reliable Distributed Systems. Journal of the ACM, 43(2):225-267, 1996.

[3] Chandra T., Hadzilacos V. and Toueg S., The Weakest Failure Detector for Solving Consensus. Journal of the ACM, 43(4):685-722, 1996.

[4] Chaudhuri S., More Choices Allow More Faults: Set Consensus Problems in Totally Asynchronous Systems. Information and Computation, 105:132-158, 1993.

[5] Fischer M.J., Lynch N.A. and Paterson M.S., Impossibility of Distributed Consensus with One Faulty Process. Journal of the ACM, 32(2):374-382, 1985.

[6] Guerraoui R., Kapałka M. and Kouznetsov P., The Weakest Failure Detectors to Boost Obstruction-Freedom. Proc. 20th Symposium on Distributed Computing (DISC'06), Springer Verlag LNCS \#4167, pp. 376-390, Stockholm (Sweden), 2006.

[7] Guerraoui R. and Raynal M., The Information Structure of Indulgent Consensus. IEEE Transactions on Computers. 53(4):453-466, 2004.

[8] Guerraoui R. and Raynal M., The Alpha of Indulgent Consensus. The Computer Journal. To appear, 2006.

[9] Herlihy M.P., Wait-Free Synchronization. ACM Transactions on Programming Languages and Systems, 13(1):124-149, 1991.

[10] Herlihy M.P. and Penso L. D., Tight Bounds for $k$-Set Agreement with Limited Scope Accuracy Failure Detectors. Distributed Computing, 18(2): 157-166, 2005.

[11] Herlihy M.P. and Shavit N., The Topological Structure of Asynchronous Computability. Journal of the ACM, 46(6):858-923,, 1999.

[12] Lamport L., The Part-Time Parliament. ACM Transactions on Computer Systems, 16(2):133-169; 1998.

[13] Loui M.C., Abu-Amara H., Memory requirements for agreement among unreliable asynchronous processes. Advances in Computing research, JAI Press, 4:163-183, 1987.

[14] Mostéfaoui A., Rajsbaum S., Raynal M. and Travers C., Irreducibility and Additivity of Set Agreement-oriented Failure Detector Classes. Proc. 25th ACM Symposium on Principles of Distributed Computing PODC'06, ACM Press, Denver (Colorado), 2006.

[15] Mostéfaoui A. and Raynal M., $k$-Set Agreement with Limited Accuracy Failure Detectors. Proc. 19th ACM Symposium on Principles of Distributed Computing (PODC'00), ACM Press, pp. 143-152, 2000.

[16] Mostéfaoui A. and Raynal M., Leader-Based Consensus. Parallel Processing Letters, 11(1):95-107, 2001.

[17] Mostéfaoui A., Raynal M. and Travers C., Exploring Gafni’s reduction land: from $\Omega^{k}$ to wait-free adaptive $\left(2 p-\left\lceil\frac{p}{k}\right\rceil\right)-$ renaming via $k$-set agreement. Proc. 20th Symposium on Distributed Computing (DISC'06), Springer Verlag LNCS \#4167, pp. 1-15, Stockholm (Sweden), 2006.

[18] Neiger G., Failure Detectors and the Wait-free Hierarchy. Proc. 14th ACM Symp. on Principles of Distributed Computing (PODC'95), ACM Press, pp. 100-109, 1995.

[19] Saks M. and Zaharoglou F., Wait-Free $k$-Set Agreement is Impossible: The Topology of Public Knowledge. SIAM Journal on Computing, 29(5):1449-1483, 2000.

[20] Yang J., Neiger G. and Gafni E., Structured Derivations of Consensus Algorithms for Failure Detectors. Proc. 17th ACM Symp. on Principles of Distributed Computing (PODC'98), ACM Press, pp.297-308, 1998. 